\title{
Evaluation of LAMP for the diagnosis of Loa loa infection in dried blood spots compared to PCR-based assays and microscopy
}

\author{
Thuy-Huong Ta-Tang, ${ }^{1,6}+$, Pedro Berzosa ${ }^{1,6}$, José Miguel Rubio ${ }^{2,6}$, María Romay-Barja ${ }^{1,6}$, \\ Policarpo Ncogo ${ }^{3,4}$, Diego Agudo ${ }^{5}$, Zaida Herrador ${ }^{1,6}$, Laura Cerrada-Gálvez ${ }^{5}$, Agustín Benito, \\ ${ }^{1}$ National Centre of Tropical Medicine, Institute of Health Carlos III, Malaria and Neglected Tropical Diseases Laboratory, Madrid, Spain \\ ${ }^{2}$ National Microbiology Centre, Institute of Health Carlos III, Malaria and Emerging Parasitic Diseases Laboratory, Madrid, Spain \\ ${ }^{3}$ Ministry of Health, Malabo, Equatorial Guinea \\ ${ }^{4}$ Fundación Estatal, Salud, Infancia y Bienestar Social, Madrid, Spain \\ ${ }^{5}$ Universidad Complutense de Madrid, Facultad de Ciencias Biológicas, Madrid, Spain \\ ${ }^{6}$ Cooperative Research Network on Tropical Diseases (Red de Investigación Colaborativa en Enfermedades Tropicales), Madrid, Spain
}

BACKGROUND Loa loa is a filarial species found exclusively in West and Central Africa. Microscopy is the traditional diagnosis method for human loiasis. Several molecular methods have developed as an alternative approach for identification of $L$. loa filarial parasites.

OBJECTIVES The aim of this study was to evaluate a Loa-Loop-mediated isothermal amplification (LAMP) assay to diagnose loiasis disease on dried blood spots (DBS) samples, compared to microscopy, filaria-real time-polymerase chain reaction (PCR) and nested-Loa PCR.

METHODS A total of 100 DBS samples and 100 blood smears were used for this study. DNA was extracted using saponin/Chelex method. DNA isolated was assayed by a Loa-LAMP assay in parallel to microscopy, filaria-real time PCR and nested-Loa PCR. The sensitivities and specificities of Loa-LAMP assay was computed comparing to each one of the reference methods.

FINDINGS Loa-LAMP's sensitivity was more than $90 \%$ and specificity was nearly $100 \%$ when compared to molecular methods. On the other hand, sensitivity was decreased a bit when Loa-LAMP faced microscopy, but keeping the other statistical values high.

MAIN CONCLUSIONS Loa-LAMP is an appropriate method for loiasis diagnosis in endemic areas. Though, it has disadvantages like the reagents' high price at the moment and not to be able to detect more filarial species at once.

Key words: filariae - Loa loa - DBS - molecular diagnosis - LAMP - real-time PCR

Human filariasis is a group of diseases produced by parasitic worms called filariae. These parasites are nematodes (roundworms) and are found in different adult tissues, depending on the species..$^{(1,2,3,4,5)}$ Filarial infections are some of most prevalent infections in the tropics, and two of them, namely onchocerciasis and lymphatic filariasis (LF), are classified amongst the neglected tropical diseases (NTDs) by the World Health Organization (WHO). ${ }^{(6,7)}$

Loa loa is a human filarial species, found exclusively in Western and Central Africa and transmitted by the bite of the Chrysops deerfly. This filarial worm causes loiasis, also known as African eye worm. ${ }^{(8)}$ In several endemic areas, L. loa co-exists with Onchocerca volvulus, Wuchereria bancrofti and Mansonella perstans. ${ }^{(5,7,9,10)}$

\section{doi: 10.1590/0074-02760210210}

Financial support: Fundación Estatal, Salud, Infancia y Bienestar Social (Institute of Health Carlos III) and Cooperative Research Network on Tropical Diseases.

T-HT-T is hired at the Malaria and Neglected Tropical Diseases Laboratory of National Centre of Tropical Medicine with a contract named ISCIII-SARA BORRELL CD17CIII/00018, financed by the Institute of Health Carlos III.

+ Corresponding author: tta@isciii.es

(D) https://orcid.org/0000-0001-7058-4839

Received 16 June 2021

Accepted 14 December 2021
Although L. loa was traditionally an absent filaria for the WHO, it began to receive increasing attention when severe adverse events presenting as encephalopathy occurred in onchocerciasis control projects supported by the African Programme for Onchocerciasis Control following treatment with ivermectin. Hence, in areas where loiasis is co-endemic with onchocerciasis and/or LF, the ability to safely carry out mass drug administration with ivermectin is impeded, especially at very high microfilarial loads (> 30,000 microfilariae per millilitre (mf/ $\mathrm{mL}))^{\left({ }^{(, 11)}\right.}$ Despite the important role of L. loa in onchocerciasis and/or LF co-infections, this filarial parasite is not included in the WHO's list of NTDs. However, the Expanded Special Project for Elimination of Neglected Tropical Diseases (ESPEN), established in May 2016, which is a project dedicated to the control and elimination of the five NTDs amenable to preventive chemotherapy (LF, onchocerciasis, schistosomiasis, soil-transmitted helminthiasis and trachoma) does include L. loa in the list of diseases to be eliminated. ${ }^{(12)}$

Conventionally, laboratory diagnosis of human loiasis relies on the detection of $\mathrm{mf}$ on Giemsa-stained or haematoxylin/eosin-stained thick and/or thin blood films by microscopy, with or without prior concentration. ${ }^{(13)}$ Based on morphological descriptions, L. loa $\mathrm{mf}$ are long and thick (205-300 x 6-8 $\mu \mathrm{m})$,with a sheath and a terminal nucleus in their tail..$^{(2,4,13,14,15)}$ However, micros- 
copy has major disadvantages, as it is a time-consuming, labor intensive and tedious method. The efficacy of $\mathrm{mf}$ detection is further decreased by the long pre-patency and the possibility of mild or occult loiasis, in which the load of $\mathrm{mf}$ parasites is undetectable microscopically. ${ }^{(1,4)}$

Several polymerase chain reaction (PCR) techniques have been developed to detect Loa-filarial infection in humans, with a high accuracy for detecting single or mixed infections with other filarias. As such, they have been shown to be an excellent alternative diagnostic method to microscopy. However, PCR methods have the disadvantage of requiring expensive equipment, laboratories with good infrastructure, a reliable electrical supply and highly trained staff, in addition to a long amplification process. ${ }^{(16,17,18,19)}$

Loop-mediated isothermal amplification (LAMP) for $L$. loa pathogen has also been developed to overcome the limitations of the PCR techniques. This molecular method is relatively recent, having emerged in $2000^{(20)}$ as a field-friendly and cost-effective diagnostic tool that requires no major capital equipment, naked eye detection of amplification by turbidity, colorimetry or fluorescence with the use of an intercalating DNA dye..$^{(21,22)}$ Like real-time PCR, LAMP is a one-step amplification reaction but with the advantage of amplification under isothermal conditions. ${ }^{(23)}$

As such, the LAMP assay has emerged as a new molecular method with the potential to replace PCR methods in the near future given its numerous advantages and outstanding efficiency. ${ }^{(23,24)}$ However, its usefulness must be tested in each case since it may also have limitations that should be analysed, including the origin and conservation of the samples.

This study has been performed as the National Centre of Tropical Medicine (Madrid, Spain) carries out international scientific-technical cooperation programs with African countries where this disease is found. Recently, the Centre was awarded several projects to assess human filariasis transmission in Equatorial Guinea and develop national recommendations to move towards onchocerciasis elimination and LF/loiasis control at a country level.

The aim of this study was to evaluate the usefulness of a previously described LAMP assay to diagnose loiasis disease in stored samples, preserved as dried blood spot (DBS), compared to the reference methods used in the laboratory for the diagnosis of loiasis disease, namely microscopy, filaria-real time-PCR and nested-loa PCR.

\section{MATERIALS AND METHODS}

Clinical samples - DBS and thick and thin blood smear (on the same slide) samples were obtained from the Laboratory of the National Centre of Tropical Medicine's repository (collection number C.0005278/ISCIII/ Spain), which is registered according to the Spanish Law RD 1716/2011 (article 22. 1). Both DBS and thick and thin blood smears belonged to the malaria project PREVAMAL (2013), to provide baseline data on malaria prevalence in Equatorial Guinea. ${ }^{(25)}$ Blood samples for each participant were taken from the finger and spotted onto Whatman $903^{\mathrm{TM}}$ paper (GE Healthcare Bio-Sciences Corp.) for molecular diagnosis and onto a clean, grease-free microscope slide for microscopy diagnosis. DBS samples had been stored in double zip-lock plastic bags with silica gel absorbent at $-20^{\circ} \mathrm{C}$. Thick and thin blood smears had been stained with $10 \%$ Giemsa solution for $10 \mathrm{~min}$. Although there were more than 1700 DBS and stained thick and thin blood smears in this malaria project, only 100 paired samples were selected for this study (100 DBS samples plus their corresponding thick and thin blood smears), using an adequate preservation and very good quality of the slides, and availability of a sufficient quantity of blood on the DBS to be extracted, as the main selection criteria.

Microscopy - The thick and thin blood smears were used to characterise the $\mathrm{mf}$ and calculate the microfilaraemia taking into account that each thick and thin blood smear contained approximately 20 and $5 \mu \mathrm{L}$, respectively. Morphological identification for $\mathrm{mf}$ was performed in line with published guidelines ${ }^{(14)}$ and examined by expert microscopists from the Laboratory of the National Centre of Tropical Medicine. Microfilariae densities were expressed as microfilariae per milliliter of blood $(\mathrm{mf} / \mathrm{mL})$ under a 10x magnification, and the filarial species was determined at a 100x magnification with immersion oil. All fields were examined before declaring a slide negative. The microfilariae densities quoted are the average value for the thick and thin films by microscopic examination.

$D B S$ - Positive and negative controls were prepared using a fresh whole blood sample in EDTA, supplied by the Malaria and Emerging Parasitic Diseases Laboratory (Madrid, Spain). Thus $70 \mu \mathrm{L}$ of blood was spotted onto Whatman filter paper and the spot allowed to dry in air for one day. DBS from infected and uninfected persons was previously diagnosed by microscopy on a blood smear. For each set of extractions performed, positive and negative DBS samples, as well as a blank (filter paper without blood), were always executed in parallel with the undiagnosed samples.

DNA extraction - DNA was extracted from DBS using the classical saponin/Chelex method, with slight modifications from the original protocol. ${ }^{(26)}$ Two filter paper discs measuring $5 \mathrm{~mm}$ in diameter were punched from the center of a circle using handheld hole puncher. These discs were immediately placed into a $1.5 \mathrm{~mL}$-tube, $1 \mathrm{~mL}$ of $0.5 \%$ saponin (Fluka Biochemika. Sigma-Aldrich Chemie $\mathrm{GmbH}$ ) in autoclaved phosphate-buffered saline (PBS) 1x was added, the resulting solution mixed thoroughly 2-3 times and incubated overnight at $4^{\circ} \mathrm{C}$ or at $37^{\circ} \mathrm{C}$ for $1 \mathrm{~h}$. The brown solution obtained was aspirated and replaced with $1 \mathrm{~mL}$ of autoclaved PBS 1x; the tube was then incubated for $30 \mathrm{~min}$ at $4^{\circ} \mathrm{C}$. During this step, $100 \mathrm{~mL}$ of a solution of 5\% Chelex-100 (Bio Rad, Richmond, CA) in water was heated at $100^{\circ} \mathrm{C}$ in a magnetic stirrer. After aspiration of the PBS 1x, $200 \mu \mathrm{L}$ of hot Chelex was added to the two paper discs, vortexed at high speed for $30 \mathrm{~s}$, and placed in the heatblock at $98^{\circ} \mathrm{C}$ for 10 min with a brief vortex again, once during and once after the incubation. After centrifugation at full speed $(13,000$ rpm) for $2 \mathrm{~min}$, the supernatant was carefully recovered so as not to remove any Chelex. The isolated DNA was 
centrifuged again at full speed under the same conditions to remove any remaining Chelex and collected into a new tube. Supernatants were used for any DNA-based technique immediately, stored at $4^{\circ} \mathrm{C}$ for use in the following few days or at $-20^{\circ} \mathrm{C}$ for a long future analysis.

Filter paper samples were handled according to safety procedures to prevent contamination of samples with each other by disinfecting the handle punch in $\mathrm{NaOH} 5 \mathrm{M}$ solution and rinsing the residual $\mathrm{NaOH}$ with distilled water.

Prior to molecular testing, the isolated DNA samples should be centrifuged at $13,000 \mathrm{rpm}$ for 2 min to place the remaining Chelex at the bottom of the tube. Chelex-100 is a chelating resin with a high affinity for multivalent metal ions, so it can inhibit the PCR by binding to $\mathrm{Mg}^{2+}$ ions. ${ }^{(27)}$

The quality of the DNA obtained using the saponin/ Chelex extraction method was checked using Nested-Malaria PCR method, which uses the human small subunit ribosomal RNA gene sequences as internal control. ${ }^{(28,29)}$

Molecular methods - The presence of $L$. loa parasites in the DNA isolated was subsequently assayed using three different molecular methods, targeting different genes to diagnose loiasis infections, as indicated briefly below:

- Filaria-real time-PCR (F-RT-PCR): targeting the internal transcribed spacer one (ITS1) of the nuclear ribosomal gene of all filarial species. ${ }^{(17)}$ In this study, the annealing temperature was varied by $48^{\circ} \mathrm{C}$ and the PCR mixture used was Luna ${ }^{\circledR}$ Universal qPCR Master Mix 2x (New England Biolabs). The cycling conditions comprised an initial denaturation step at $95^{\circ} \mathrm{C}$ for $1 \mathrm{~min}$, followed by 45 cycles of denaturation at $95^{\circ} \mathrm{C}$ for $15 \mathrm{~s}$, annealing at $48^{\circ} \mathrm{C}$ for $20 \mathrm{~s}$ and extension at $60^{\circ} \mathrm{C}$ for 30 s. A sample was considered positive for filarial parasite if the melting temperature (Tm) curve of the amplified fragments was $\mathrm{Tm}=77^{\circ} \mathrm{C} \pm 0.5^{\circ} \mathrm{C}$ and the species identification was according to amplified product size after analysing in a multicapillary electrophoresis.

- Nested-Loa PCR: this nested PCR targets the gene encoding the $L$. loa $15-\mathrm{kD}$ protein. ${ }^{(18)}$ The original protocol was followed, except for the volume of DNA template: $5 \mu \mathrm{L}$ was used for the first PCR round and $2 \mu \mathrm{L}$ of the first amplification product was used for the nested PCR.

- Loa-LAMP: the isothermal amplification assay was designed to target the $L$. loa-specific gene sequences LLMF72. ${ }^{(22)}$ The LAMP reaction was performed using a WarmStart Colorimetric LAMP 2x Master Mix (New England Biolabs) DNA amplification kit in a total volume of $25 \mu \mathrm{L}(20 \mu \mathrm{L}$ of reaction mixture and $5 \mu \mathrm{L}$ of DNA template). Primer concentrations were the same as calculated in the original protocol without modifications. All reactions were performed at $65^{\circ} \mathrm{C}$ for up to 30 min and heated at $80^{\circ} \mathrm{C}$ for 5 min, inactivating the $B s t$ DNA polymerase. Positive amplification results were detected by the naked eye based on the colour change using the $\mathrm{pH}$-sensitive dye Phenol red.

For each reaction performed, appropriate positive and negative controls were always included. Positive controls were DBS samples of $L$. loa-microscopy positive individuals; negative controls were DBS samples of L. loa-microscopy negative individuals from filarialendemic regions who were negative for filarial infection but could be positive or negative for other tropical parasites, such as Plasmodium spp.

All samples were run in duplicate. If the duplicates varied (one positive, one negative), the samples were rerun in triplicate.

All F-RT-PCR reactions were performed using a Rotor Gene Q 5plex (QIAGEN GMBH, Germany). The remaining DNA amplifications, including the LoaLAMP, were performed using an Applied Biosystems GeneAmp ${ }^{\circledR}$ PCR System 2700 . Amplification products were detected using QIAxcel Advanced (QIAGEN GMBH, Germany), an automated system for analysing DNA fragments based on multicapillary electrophoresis.

See Table I for a more detailed overview of primer sequences, target gene, product size and other characteristics of the assays.

DNA sequencing and sequence analysis - All the products amplified by F-RT-PCR and Nested-Loa PCR techniques were purified using Speedtools PCR Clean Up Kit 250 rxns (Biotools, B\&M Lab, SA), then sequenced in both directions using Big Dye Terminator v3.1 Cycle Sequencing in an ABI PRISM ${ }^{\circledR} 3700$ DNA Analyzer. Sequences were compared to the genbank database using basic local alignment search tool (BLAST) ${ }^{(30)}$ and aligned using CLUSTAL $\mathrm{W}^{(31)}$ to confirm the diagnosis and detect possible mutations.

In the case of the Loa-LAMP method, the positive post-LAMP reaction mixture subjected to gel electrophoresis produces many bands of different sizes in a reproducible ladder-like pattern and these products could not be sequenced.

Statistical analysis - Statistical values [sensitivity, specificity, positive predictive value (PPV), negative predictive value (NPV) and kappa index] for the LoaLAMP assay was calculated compared to each of the reference methods using the free software WinEpi: Working in Epidemiology. ${ }^{(32)}$ The confidence intervals (CI) were established at $95 \%$.

\section{RESULTS}

Overall, 66 samples were identified as negative by all the tests and 34 samples were positive in at least one of the methods tested (microscopy or molecular test). A list of positive samples has been created to facilitate the following and understanding of the results (Table II).

Microscopy - Out of 100 thick and thin blood smears analysed by microscopy, 73 (73\%) were negative and 27 (27\%) were positive: 13 were L. loa, $11 \mathrm{M}$. perstans and 3 were mixed infections (L. loa + M. perstans). The average microfilaremia for $L$. loa was 3047 $\mathrm{mf} / \mathrm{mL}$ (parasite count range 200-12200 $\mathrm{mf} / \mathrm{mL}$ ) and for $M$. perstans was $764 \mathrm{mf} / \mathrm{mL}$ (parasite count range $100-3200 \mathrm{mf} / \mathrm{mL}$ ) (Table II).

$F-R T-P C R$ - This method was able to detect $30(30 \%)$ positive samples: $11 \mathrm{~L}$. loa, $17 \mathrm{M}$. perstans and two mixed infections. Seven M. perstans-F-RT-PCR positive samples were missed by microscopy, whereas four microscopy positive samples were missed by F-RT-PCR ( 2 L. loa, 1 M. perstans and 1 mixed infection) (Table II). 


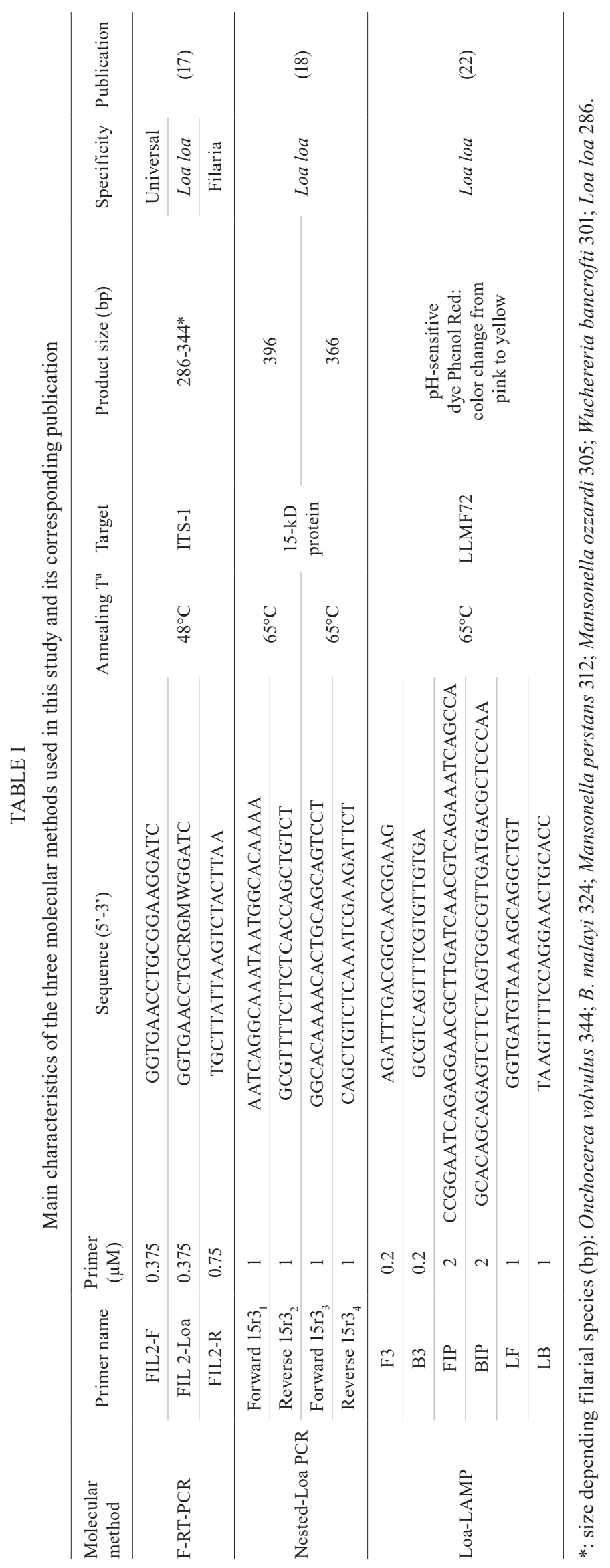


TABLE II

List of positive samples with their corresponding results obtained by microscopy and molecular methods

\begin{tabular}{|c|c|c|c|c|c|}
\hline Samples & Microscopy & Microfilaremia $(\mathrm{mf} / \mathrm{mL})$ & F-RT-PCR & NESTED-LOA PCR & LOA-LAMP \\
\hline 34 & $\mathrm{~L}$ & 1100 & $\mathrm{~L}$ & $\mathrm{~L}$ & $\mathrm{~L}$ \\
\hline 57 & $\mathrm{~L}$ & 300 & $\mathrm{~L}$ & $\mathrm{~L}$ & $\mathrm{~L}$ \\
\hline 65 & $\mathrm{~L}$ & 500 & $\mathrm{~L}$ & $\mathrm{~L}$ & $\mathrm{~L}$ \\
\hline 90 & $\mathrm{~L}$ & 2200 & $\mathrm{~L}$ & $\mathrm{~L}$ & $\mathrm{~L}$ \\
\hline 98 & $\mathrm{~L}$ & 3600 & $\mathrm{~L}$ & $\mathrm{~L}$ & $\mathrm{~L}$ \\
\hline 144 & $\mathrm{~L}$ & 12200 & $\mathrm{~L}$ & $\mathrm{~L}$ & $\mathrm{~L}$ \\
\hline 169 & $\mathrm{~L}$ & 400 & $\mathrm{~N}$ & $\mathrm{~N}$ & $\mathrm{~N}$ \\
\hline 179 & $\mathrm{~L}$ & 500 & $\mathrm{~L}$ & $\mathrm{~N}$ & $\mathrm{~N}$ \\
\hline 220 & $\mathrm{~L}$ & 2000 & $\mathrm{~L}$ & $\mathrm{~L}$ & $\mathrm{~L}$ \\
\hline 254 & $\mathrm{~L}$ & 11600 & $\mathrm{~L}$ & $\mathrm{~L}$ & $\mathrm{~L}$ \\
\hline 297 & $\mathrm{~L}$ & 450 & $\mathrm{~N}$ & $\mathrm{~N}$ & $\mathrm{~N}$ \\
\hline 301 & $\mathrm{~L}$ & 5600 & $\mathrm{~L}$ & $\mathrm{~L}$ & $\mathrm{~L}$ \\
\hline 319 & $\mathrm{~L}$ & 1900 & $\mathrm{~L}$ & $\mathrm{~L}$ & $\mathrm{~L}$ \\
\hline 79 & $\mathrm{Mp}$ & 200 & $\mathrm{~N}$ & $\mathrm{~N}$ & $\mathrm{~N}$ \\
\hline 133 & $\mathrm{Mp}$ & 600 & $\mathrm{Mp}$ & $\mathrm{N}$ & $\mathrm{N}$ \\
\hline 149 & $\mathrm{Mp}$ & 800 & $\mathrm{Mp}$ & $\mathrm{N}$ & $\mathrm{N}$ \\
\hline 164 & $\mathrm{Mp}$ & 100 & $\mathrm{Mp}$ & $\mathrm{N}$ & $\mathrm{N}$ \\
\hline 176 & $\mathrm{Mp}$ & 100 & $\mathrm{Mp}$ & $\mathrm{N}$ & $\mathrm{N}$ \\
\hline 192 & $\mathrm{Mp}$ & 1300 & $\mathrm{Mp}$ & $\mathrm{N}$ & $\mathrm{N}$ \\
\hline 306 & $\mathrm{Mp}$ & 3200 & $\mathrm{Mp}$ & $\mathrm{N}$ & $\mathrm{N}$ \\
\hline 308 & $\mathrm{Mp}$ & 100 & $\mathrm{Mp}$ & $\mathrm{N}$ & $\mathrm{N}$ \\
\hline 310 & $\mathrm{Mp}$ & 400 & $\mathrm{Mp}$ & $\mathrm{N}$ & $\mathrm{N}$ \\
\hline 326 & $\mathrm{Mp}$ & 1000 & $\mathrm{Mp}$ & $\mathrm{N}$ & $\mathrm{N}$ \\
\hline 341 & $\mathrm{Mp}$ & 1000 & $\mathrm{Mp}$ & $\mathrm{N}$ & $\mathrm{N}$ \\
\hline 194 & $\mathrm{~L}+\mathrm{Mp}$ & $200 ; 200$ & $\mathrm{~N}$ & $\mathrm{~N}$ & $\mathrm{~N}$ \\
\hline 276 & $\mathrm{~L}+\mathrm{Mp}$ & $200 ; 200$ & $\mathrm{~L}+\mathrm{Mp}$ & $\mathrm{L}$ & $\mathrm{L}$ \\
\hline 318 & $\mathrm{~L}+\mathrm{Mp}$ & $6000 ; 1500$ & $\mathrm{~L}+\mathrm{Mp}$ & $\mathrm{L}$ & $\mathrm{L}$ \\
\hline 74 & $\mathrm{~N}$ & 0 & $\mathrm{Mp}$ & $\mathrm{N}$ & $\mathrm{N}$ \\
\hline 76 & $\mathrm{~N}$ & 0 & $\mathrm{Mp}$ & $\mathrm{N}$ & $\mathrm{N}$ \\
\hline 104 & $\mathrm{~N}$ & 0 & $\mathrm{Mp}$ & $\mathrm{N}$ & $\mathrm{N}$ \\
\hline 106 & $\mathrm{~N}$ & 0 & $\mathrm{Mp}$ & $\mathrm{N}$ & $\mathrm{N}$ \\
\hline 137 & $\mathrm{~N}$ & 0 & $\mathrm{Mp}$ & $\mathrm{N}$ & $\mathrm{L}$ \\
\hline 138 & $\mathrm{~N}$ & 0 & $\mathrm{Mp}$ & $\mathrm{N}$ & $\mathrm{N}$ \\
\hline 141 & $\mathrm{~N}$ & 0 & $\mathrm{Mp}$ & $\mathrm{N}$ & $\mathrm{N}$ \\
\hline
\end{tabular}

F-RT-PCR: Filaria-real time-polymerase chain reaction; L: Loa loa; Mp: Mansonella perstans; N: negative. For a better visualisation of the results, each type of infection has a different padding. Microfilaremia in mixed infections: the first value is for $L$. loa, the second value is for M. perstans.

Nested-Loa PCR - This method detected 12 (12\%) L. loa positives and 88 (88\%) L. loa negatives (Table II). Four samples $L$. loa-microscopy positives ( 3 L. loa single and 1 mixed infection) were missed by Nested-Loa PCR.

Loa-LAMP - This method detected 13 (13\%) L. loa positives and $87(87 \%)$ L. loa negatives, and missed the same four samples as Nested-Loa PCR (Table II). A sample was considered positive for L. loa if the color changed from pink to yellow (Figure).

Statistical analysis - Loa-LAMP's sensitivity was more than $90 \%$ and the specificity was nearly $100 \%$ when compared to molecular methods (F-RT-PCR and Nested-Loa PCR). The concordance (kappa index) was good or excellent. On the other hand, sensitivity (75\%) 


\section{$\begin{array}{lllllllllllll}34 & 57 & 65 & 90 & 98 & 144 & 220 & 254 & \text { PC } & 79 & 133 & 149 & \text { NC NTC }\end{array}$}

Loa-LAMP assay: DBS samples with their corresponding number assigned in the laboratory. The WarmStart Colorimetric LAMP 2x Master Mixes contains the $\mathrm{pH}$-sensitive dye Phenol Red that changes color from bright pink (negative amplification for $L$. loa) to yellow (positive amplification for $L$. loa) as shown here after amplification for 30 minutes of. PC: positive control (positive for L. loa by microscopy and F-RT-PCR). NC: negative control (negative for any filarial parasite); NTC: non-template control.

was slightly lower when comparing Loa-LAMP with microscopy, although the other statistical values remained high (Table III).

\section{DISCUSSION}

The L. loa parasite is no longer as insignificant as it used to be in the past and its growing importance is due to the marked negative effect caused in people who live in co-endemic areas with $O$. volvulus and $W$. bancroft $i$, where mass drug administration is currently underway. $(8,18,22)$ As the goal of ESPEN is to accelerate elimination of the five most prevalent NTDs (LF, onchocerciasis, soil-transmitted helminthiases, schistosomiasis and trachoma) accurate $L$. loa parasite detection is essential for addressing the problem where $O$. volvulus, $W$. bancroft $i$ and $L$. loa infections are co-endemic to achieve global elimination goals for $O$. volvulus and $W$. bancrofti. ${ }^{(7,12)}$

Microscopy on stained thick and/or thin blood smears remains the gold standard for routine clinical diagnosis of human loiasis in remote areas, due to the low cost of the materials, the ease with which it is performed, the minimal infrastructure required, and the ability to quantify the microfilaremia. However, this approach has limited sensitivity and specificity, and is not suitable for large-scale $\mathrm{mf}$ screening in disease-endemic areas. ${ }^{(33,34)}$

In the few last decades, several molecular methods, such as real-time PCR, Nested-PCR and LAMP, have been reported for the diagnosis of human loiasis infection with high sensitivity and specificity. ${ }^{(16,17,18,22)}$ These methods can easily diagnose infection by $L$. loa even if the parasite cannot be detected microscopically.

In this study, the utility of a Loa-LAMP method, developed by Drame et al. in 2014, ${ }^{(2)}$ was evaluated and compared to microscopy and two validated and wellknown molecular methods, namely F-RT-PCR ${ }^{(17)}$ and Nested-Loa PCR, ${ }^{(18)}$ on 100 DBS. As F-RT-PCR and Nested-Loa PCR are not convenient methods for L. loa pathogen detection in the field and at a point-of-care setting, a Loa-LAMP assay was evaluated in light of the very promising results obtained by Drame et al. ${ }^{(22)}$

Loa-LAMP and the two other PCR assays described previously were developed for different targets of the parasite, and each one is probably represented by different numbers of copies in the filarial genome. These three selected methods targeting different genes might be an advantage, since if one clinical sample is positive for L. loa by all three methods, this confirms that the patient had loiasis infection.
A good diagnostic test should have a high validity, expressed as a function of sensitivity and specificity, which means that the percentages of false-positive and false-negative results should be limited, and that the different targets would have no influence on the statistical values. To calculate the relative sensitivity and specificity of the Loa-LAMP test, microscopy, F-RT-PCR and Nested-Loa PCR were taken as the standard test, with an estimated validity of approximately $100 \%$.

The samples that were $L$. loa-microscopy single-positive but were missed by molecular methods may have had a very low $\mathrm{mf}$ load $(\leq 500 \mathrm{mf} / \mathrm{mL})$. Blood samples with very low microfilaremia have a lower possibility of taking one microfilariae in the punch because the distribution of $\mathrm{mf}$ on the blood spot is not homogenous. Thus, parasite material seems to be less concentrated towards the extreme edges of the blood spot, as shown in the experiment carried out by Baidjoe et al. ${ }^{(35)}$ Among the three mixed infections (L. loa + M. perstans) detected by microscopy, no loa parasite could be detected in one sample by any of the three molecular methods. This mixed infection also had a very low microfilaremia $(200 \mathrm{mf} / \mathrm{mL})$.

False-negative results due to the presence of potential enzyme inhibitors in the samples, or the poor DNA quality or concentration yielded by the saponin/Chelex DNA extraction method, were discarded, since all of them were checked with an internal extraction control present in the Nested-Malaria PCR method. ${ }^{(28,29)}$

Samples infected by $M$. perstans were also included in this study to identify possible cross-reactivity with $M$. perstans using the Loa-LAMP. There was only one case (\#137) that tested M. perstans-F-RT-PCR positive and positive for L. loa by Loa-LAMP. This could be a possible mixed infection in which each method was able to detect the filarial parasite for which it is most sensitive. Overall, Loa-LAMP did not exhibit a cross-reaction with $M$. perstans DNA, although the possibility of a cross-reaction cannot be excluded.

Only one $M$. perstans-microscopy positive (\#79) was missed by F-RT-PCR, probably because this sample had $200 \mathrm{mf} / \mathrm{mL}$. Again, the sensitivity of the molecular method appeared to be compromised because of the low microfilaremia and the lower probability of collecting the microfilariae. It should be noted that FRT-PCR detected seven more $M$. perstans which were not detected by microscopy.

After evaluating the Loa-LAMP assay, it has been found that the sensitivity and specificity are very high and the concordance between methods shows excellent agreement according to the kappa index values, thus suggesting that the method may be suitable for use with clinical samples in resource-limited areas endemic for loiasis.

As can be seen by the results, the Loa-LAMP assay may also be very useful as a potential point-of-care tool for the rapid amplification and easy detection of $L$. loa DNA in the field. Indeed, its statistical values are very similar to those found for F-RT-PCR and Nested-Loa PCR when tested with the same clinical samples.

Despite using a different source of samples, another method for DNA isolation and different reagents, the results obtained from the colorimetric LAMP assays in 
TABLE III

Statistical values obtained for Loa-LAMP assay comparing to microscopy, Filaria-real time-polymerase chain reaction (F-RT-PCR) and Nested-Loa PCR, references methods used in the laboratory

\begin{tabular}{ccccc}
\hline & & Microscopy & F-RT-PCR & Nested-Loa PCR \\
\hline & Sensitivity \% (95\% CI) & $75.0 \%(53.8 \%, 96.2 \%)$ & $92.3 \%(77.8 \%, 106.8 \%)$ & $100.0 \%(100.0 \%, 100.0 \%)$ \\
\cline { 2 - 5 } Loa-LAMP & Specificity \% (95\% CI) & $98.8 \%(96.5 \%, 101.1 \%)$ & $98.9 \%(96.6 \%, 101.1 \%)$ & $98.9 \%(96.6 \%, 101.1 \%)$ \\
\cline { 2 - 5 } & PPV \% (95\% CI) & $92.3 \%(77.8 \%, 106.8 \%)$ & $92.3 \%(77.8 \%, 106.8 \%)$ & $92.3 \%(77.8 \%, 106.8 \%)$ \\
\cline { 2 - 5 } & NPV \% (95\% CI) & $95.4 \%(91.0 \%, 99.8 \%)$ & $98.9 \%(96.6 \%, 101.1 \%)$ & $100.0 \%(100.0 \%, 100.0 \%)$ \\
\cline { 2 - 5 } & Kappa index \% (95\% CI) & $79.9 \%(62.7 \%, 97.1 \%) *$ & $91.2 \%(79.0 \%, 103.3 \%) * *$ & $95.4 \%(86.5 \%, 104.3 \%) * *$ \\
\hline
\end{tabular}

PPV: positive predictive value; NPV: negative predictive value; CI: confidence intervals; *: good agreement; **: excellent agreement.

this study are similar to those reported by Drame et al., ${ }^{(22)}$ with a high positive-predictive $92.3 \%$ (95\% CI 77.8\%$106.8 \%$ ) and high negative-predictive [range 95.4\% (95\% CI $91 \%-99.8 \%)-100.0 \%$ (100.0\%-100.0\%), depending on the method used for comparison].

In our opinion, the major limitation of the LAMP assay is the price of the kit used. Thus, in our study, the cost per sample was 3.5-times higher than for real-time PCR and almost five-times higher than for Nested-Loa PCR. As such, this could be a major limitation for its use in developing countries. In addition, the Loa-LAMP method still needs electrical power for the water bath or heating block to carry out the reaction, and a freezer is needed to store the Loa-LAMP reagents, and not all the endemic areas have a continuous power supply.

Another disadvantage of this Loa-LAMP technique is that it is monoplex and, therefore, has a limited capacity to detect a wide variety of filarial species at once. Indeed, it has been specifically designed to detect L. loa DNA even though an absence of $L$. loa DNA does not rule out the presence of other blood microfilariae ( $M$. perstans or $W$. bancrofti).

In conclusion - As illustrated by the results from this study, Loa-LAMP is very similar in terms of sensitivity and specificity to established diagnostic methods for detecting loiasis infection, such as microscopy, F-RT-PCR and Nested-Loa PCR. As such, it is an appropriate method for loiasis diagnosis if a rapid screening of patients in endemic areas is required. Moreover, Loa-LAMP is a good alternative for use in the field, as no major capital equipment is required, the turnaround times are short compared to F-RT-PCR and Nested-Loa PCR, and amplification by colorimetry can be detected by the naked eye, although the main disadvantages are the high price of reagents and the inability to detect more than one filarial species simultaneously. Given the good results for the diagnosis of LAMP, its applicability in the field and the ease with which positives can be detected, laboratories such as ours should design a LAMP that can detect more than one species simultaneously in order to facilitate work in the field. The basis of good scientific/ technical cooperation, one of the responsibilities of our Centre, is to be able to offer endemic areas fast and reliable diagnostic tools that facilitate diagnosis, so that patients can quickly receive effective treatment.
Ethics approval and consent to participate - All the clinical samples, DBS and blood smears, had the authorisation of the director of National Centre of Tropical Medicine to be used in this study with the unique purpose to compare the Loa-LAMP assay to microcopy, and two molecular techniques, filaria-real time-PCR and nested-loa PCR.

\section{List of abbreviations}

BLAST: Basic Local Alignment Search Tool; bp: basepairs; ${ }^{\circ} \mathrm{C}$ : degrees Celsius; $\mathrm{CI}$ : confidence intervals; DBS: dried blood spots; DNA: deoxyribonucleic acid; EDTA: ethylene diamine tetra-acetic acid; ESPEN: Expanded Special Project for Elimination of Neglected Tropical Diseases; ITS1: internal transcribed spacer one; LAMP: Loop-mediated isothermal amplification; LF: lymphatic filariasis; F-RT-PCR: filaria-real timePCR; mf: microfilariae; mL: milliliter; mm: millimeter; min: minutes; NPV: negative predictive value; NTDs: neglected tropical diseases; PBS: phosphate-buffered saline; PCR: polymerase chain reaction; PPV: positive predictive value; rDNA: ribosomal deoxyribonucleic acid; RNA: ribonucleic acid; rpm: revolutions per minute; WHO: World Health Organization; s: seconds; Tm: melting temperature; $\mu \mathrm{L}$ : microliter.

\section{AUTHORS' CONTRIBUTION}

THTT, PB and JMR conceived and designed the research, and drafted the manuscript with input from all other authors; THTT, DA and LCG performed the acid nucleic isolation and molecular techniques; THTT, PN, DA and AB carried out microfilariae microscopic diagnosis; THTT performed data processing and analyses; MRB analysed and interpreted statistical data; MRB, AB and ZH critically revised the manuscript for intellectual content. All authors reviewed and approved the final manuscript prior to submission. The authors declare that there is no conflict of interest regarding the publication of this article.

\section{REFERENCES}

1. Nanduri J, Kazura JW. Clinical and laboratory aspects of filariasis. Clin Microbiol Rev. 1989; 2(1): 39-50.

2. Walther M, Muller R. Diagnosis of human filariases (except onchocerciasis). Adv Parasitol. 2003; 53: 149-93.

3. Alhassan A, Li Z, Poole CB, Carlow CKS. Expanding the MDx toolbox for filarial diagnosis and surveillance. Trends Parasitol. 2015; 31(8): 391-400. 
4. Knopp S, Steinmann P, Hatz C, Keiser J, Utzinger J. Nematode infections: filariases. Infect Dis Clin North Am. 2012; 26(2): 359-81.

5. Cobo F. 7 - Filariasis. In: Cobo F, editor. Imported infectious diseases. Woodhead Publishing; 2014. p. 91-114. Available from: https://www.sciencedirect.com/science/article/pii/ B9781907568572500076.

6. Taylor EM. NTD diagnostics for disease elimination: a review. Diagnostics (Basel). 2020; 10(6): 375.

7. WHO - World Health Organization. Neglected tropical diseases. World Health Organization. Available from: https://www.who.int/ neglected_diseases/diseases/en/.

8. Whittaker C, Walker M, Pion SDS, Chesnais CB, Boussinesq M, Basáñez M-G. The population biology and transmission dynamics of Loa loa. Trends Parasitol. 2018; 34(4): 335-50.

9. Ta-Tang T-H, Crainey JL, Post RJ, Luz SL, Rubio JM. Mansonellosis: current perspectives. Res Rep Trop Med. 2018; 9: 9-24.

10. Ta-Tang T-H, Luz SLB, Crainey JL, Rubio JM. An overview of the management of mansonellosis. Res Rep Trop Med. 2021; 12: 93-105.

11. Molyneux DH. Filaria control and elimination: diagnostic, monitoring and surveillance needs. Trans R Soc Trop Med Hyg. 2009; 103(4): 338-41.

12. ESPEN - Expanded Special Project for Elimination of Neglected Tropical Diseases. Loiasis. Available from: https://espen.afro.who. int/diseases/loiasis.

13. Mathison BA, Couturier MR, Pritt BS. Diagnostic identification and differentiation of microfilariae. J Clin Microbiol. 2019; 57(10): e00706-19.

14. Eberhard ML, Lammie PJ. Laboratory diagnosis of filariasis. Clin Lab Med. 1991; 11(4): 977-1010.

15. Lok JB, Walker ED, Scoles GA. Filariasis. In: Eldridge BF, Edman JD, editors. Medical entomology: a textbook on public health and veterinary problems caused by arthropods. Dordrecht: Springer Netherlands; 2000; p. 299-375. Available from: https:// doi.org/10.1007/978-94-011-6472-6 9.

16. Tang T-HT, López-Vélez R, Lanza M, Shelley AJ, Rubio JM, Luz SLB. Nested PCR to detect and distinguish the sympatric filarial species Onchocerca volvulus, Mansonella ozzardi and Mansonella perstans in the Amazon Region. Mem Inst Oswaldo Cruz. 2010; 105(6): 823-8.

17. Ta T-H, Moya L, Nguema J, Aparicio P, Miguel-Oteo M, Cenzual $\mathrm{G}$, et al. Geographical distribution and species identification of human filariasis and onchocerciasis in Bioko Island, Equatorial Guinea. Acta Trop. 2018; 180: 12-7.

18. Touré FS, Kassambara L, Williams T, Millet P, Bain O, Georges AJ, et al. Human occult loiasis: improvement in diagnostic sensitivity by the use of a nested polymerase chain reaction. Am J Trop Med Hyg. 1998; 59(1): 144-9.

19. Nuchprayoon S, Junpee A, Poovorawan Y, Scott AL. Detection and differentiation of filarial parasites by universal primers and polymerase chain reaction-restriction fragment length polymorphism analysis. Am J Trop Med Hyg. 2005; 73(5): 895-900.

20. Notomi T, Okayama H, Masubuchi H, Yonekawa T, Watanabe K, Amino N, et al. Loop-mediated isothermal amplification of DNA. Nucleic Acids Res. 2000; 28(12): E63.
21. Poole CB, Sinha A, Ettwiller L, Apone L, McKay K, Panchapakesa $\mathrm{V}$, et al. In silico identification of novel biomarkers and development of new rapid diagnostic tests for the filarial parasites Mansonella perstans and Mansonella ozzardi. Sci Rep. 2019; 9(1): 10275.

22. Drame PM, Fink DL, Kamgno J, Herrick JA, Nutman TB. Loopmediated isothermal amplification for rapid and semiquantitative detection of Loa loa infection. J Clin Microbiol. 2014; 52(6): 2071-7.

23. Abdul-Ghani R, Al-Mekhlafi AM, Karanis P. Loop-mediated isothermal amplification (LAMP) for malarial parasites of humans: would it come to clinical reality as a point-of-care test? Acta Trop. 2012; 122(3): 233-40.

24. Zhang X, Lowe SB, Gooding JJ. Brief review of monitoring methods for loop-mediated isothermal amplification (LAMP). Biosens Bioelectron. 2014; 61: 491-9.

25. Berzosa P, Esteban-Cantos A, García L, González V, Navarro M, Fernández T, et al. Profile of molecular mutations in pfdhfr, pfdhps, pfmdrl, and pfert genes of Plasmodium falciparum related to resistance to different anti-malarial drugs in the Bata District (Equatorial Guinea). Malar J. 2017; 16(1): 28.

26. Plowe CV, Djimde A, Bouare M, Doumbo O, Wellems TE. Pyrimethamine and proguanil resistance-conferring mutations in Plasmodium falciparum dihydrofolate reductase: polymerase chain reaction methods for surveillance in Africa. Am J Trop Med Hyg. 1995; 52(6): 565-8.

27. Tani H, Tada Y, Sasai K, Baba E. Improvement of DNA extraction method for dried blood spots and comparison of four PCR methods for detection of Babesia gibsoni (Asian genotype) infection in canine blood samples. J Vet Med Sci. 2008; 70(5): 461-7.

28. Ta TH, Hisam S, Lanza M, Jiram AI, Ismail N, Rubio JM. First case of a naturally acquired human infection with Plasmodium cynomolgi. Malar J. 2014; 13: 68

29. Miguel-Oteo M, Jiram AI, Ta-Tang TH, Lanza M, Hisam S, Rubio JM. Nested multiplex PCR for identification and detection of human Plasmodium species including Plasmodium knowlesi. Asian Pac J Trop Med. 2017; 10(3): 299-304.

30. Altschul SF, Gish W, Miller W, Myers EW, Lipman DJ. Basic local alignment search tool. J Mol Biol. 1990; 215(3): 403-10.

31. Thompson JD, Higgins DG, Gibson TJ. CLUSTAL W: improving the sensitivity of progressive multiple sequence alignment through sequence weighting, position-specific gap penalties and weight matrix choice. Nucleic Acids Res. 1994; 22(22): 4673-80.

32. Win Epi. Working in epidemiology. Available from: http://winepi.net/.

33. Pion SD, Montavon C, Chesnais CB, Kamgno J, Wanji S, Klion $\mathrm{AD}$, et al. Positivity of antigen tests used for diagnosis of lymphatic filariasis in individuals without Wuchereria bancrofti infection but with high Loa loa microfilaremia. Am J Trop Med Hyg. 2016; 95(6): 1417-23.

34. Wanji S, Ndongmo WPC, Fombad FF, Kengne-Ouafo JA, Njouendou AJ, Tchounkeu YFL, et al. Impact of repeated annual community directed treatment with ivermectin on loiasis parasitological indicators in Cameroon: implications for onchocerciasis and lymphatic filariasis elimination in areas co-endemic with Loa loa in Africa. PLoS Negl Trop Dis. 2018; 12(9): e0006750.

35. Baidjoe A, Stone W, Ploemen I, Shagari S, Grignard L, Osoti V, et al. Combined DNA extraction and antibody elution from filter papers for the assessment of malaria transmission intensity in epidemiological studies. Malar J. 2013; 12: 272. 